\title{
Sintonía humana con los principios ecológicos: Un marco conceptual para el desarrollo sustentable
}

\section{Human attunement with ecological principles: A conceptual framework for sustainable development}

\author{
Fernanda Inéz García Vázquez \\ Víctor Corral Verdugo \\ Universidad de Sonora
}

\begin{abstract}
Resumen
Diversas teorías afirman que existen similitudes entre las comunidades humanas y los ecosistemas, y que de éstos últimos podemos aprender cómo vivir sustentablemente; sin embargo, a nivel conductual la posible relación entre la práctica de conductas sustentables y el vivir de acuerdo con los principios ecológicos ha sido poco estudiada. El presente artículo aborda dicha potencial relación, a partir de la siguiente idea: para actuar en concordancia con los principios ecológicos, es necesario conocerlos, pero también desplegar afinidad por ellos, es decir, valorarlos positivamente, para después aplicarlos, comportándonos de manera sustentable. A esta posible disposicionalidad psicológica la hemos denominado Sintonía Humana con los Principios Ecológicos (SHPE), la cual implicaría la existencia de una predisposición humana para conocer, valorar y aplicar esos principios. Con el objetivo de fundamentar la idea, en el presente trabajo se revisa la literatura relevante, en busca de indicios que apoyen la hipótesis de la existencia de la SHPE, describiendo con detalle cada uno de los principios ecológicos, explicando su relación con el desarrollo sustentable y la manera en que se podrían abordar estos principios desde la psicología.

Palabras clave: principios ecológicos, conducta sustentable, psicología ambiental, sustentabilidad.
\end{abstract}

Nota del autor

Fernanda Inez García Vázquez, Departamento de Psicología, Universidad de Sonora; Víctor Corral Verdugo, Departamento de Psicología, Universidad de Sonora.

Esta investigación está subvencionada por CONACyT (proyecto 179886).

La correspondencia en relación con este artículo debe dirigirse a Víctor Corral Verdugo, Departamento de Psicología, Universidad de Sonora, Hermosillo, Sonora, 83000.

Dirección electrónica: victorcorral@sociales.uson.mx 


\begin{abstract}
A number of theories claim that similarities exist between human communities and ecosystems, and that from them we can learn how to live sustainably. Yet, at the behavioral level, the relationship between practicing sustainable behaviors and living in accordance with ecological principles has been barely studied. This paper addresses such potential relationship, based on the idea that acting in accordance with ecological principles requires the knowledge of them but also an affinity towards those principles, and behaving accordingly (i.e., acting sustainably). We call this hypothetical relation the Human Attunement with Ecological Principles (HAEP), and assume that it implies the presence of a human predisposition to knowing, valuing and applying these principles. In developing this idea, the paper reviews relevant literature, aiming to find evidence that supports the HAEP hypothesis, describing in detail every ecological principle, explaining its relationship with sustainable development and how those principles might be addressed from a psychological standpoint.
\end{abstract}

Keywords: ecological principles, sustainable behavior, environmental psychology, sustainability.

Los seres humanos hemos utilizado $\mathrm{y}$ transformado los ecosistemas de la Tierra gracias a los avances de la tecnología y la biotecnología, para resolver las demandas crecientes de recursos, sobre todo en los últimos 50 años. Esta transformación aportó considerables beneficios al bienestar humano y al desarrollo económico, sin embargo, en los últimos años se han manifestado los costos asociados con dichos beneficios, sobre todo en cuanto a escasez de recursos, pero también en cuanto a la degradación de los procesos reguladores: la purificación del aire y agua, la regulación del clima regional y local, los riesgos naturales y las epidemias regionales y planetarias (OnaindiaOlalde, 2007).

Existe un conflicto fundamental entre el buen funcionamiento de la biósfera y la continua expansión de los sistemas económicos creados por el ser humano. Las leyes biológicas no cambiarán a pesar de todo el desarrollo tecnológico que hombres y mujeres sean capaces de lograr. Por lo tanto, resulta claro que es el sistema económico en expansión el que debe cambiar; para lograr esto podemos ser proactivos en el cambio, revisando nuestra comprensión del mundo adoptando políticas que reflejen una visión ecológica. Con lo anterior, se ayudaría a la evolución cultural, pasando de los actuales sistemas depredadores dominantes actuales a culturas más sustentables (Gowdy \& McDaniel, 1995).

Los ecosistemas globales no son capaces de soportar el estilo de vida dominante de los países industrializados. Por consecuencia, los nuevos modelos de desarrollo tienen que incluir tanto a los países desarrollados como a los más pobres. Es necesario tomar en cuenta que "crecimiento" significa un incremento material, mientras que "desarrollo" implica un cambio cualitativo, además, ambos obedecen a diferentes principios. Existen límites al crecimiento material pero no 
necesariamente límites al desarrollo. La pregunta clave, entonces es: ¿Cuáles son los factores que nos llevan al sendero de la insustentabilidad y cuáles son los caminos hacia un desarrollo sustentable? (Kammerbauer, 2001).

\section{Las conductas sustentables}

Brundtland (1987) define el desarrollo sustentable como un desarrollo susceptible de satisfacer las necesidades de la generación actual sin comprometer las posibilidades para las generaciones futuras de satisfacer las suyas. De acuerdo con lo expresado anteriormente, una manera de avanzar hacia la especificación del concepto de sustentabilidad consiste en delimitar los ejes o las dimensiones fundamentales que lo caracterizan.

Para Díaz y Spiaggi (2007) la sustentabilidad comprende varias dimensiones: La dimensión ecológica se refiere a considerar y respetar los ciclos naturales, la productividad propia de la naturaleza, la disponibilidad y finitud de los recursos, y la biodiversidad. La dimensión social implica la justicia social (para el presente $\mathrm{y}$ para las generaciones futuras), la equidad entre géneros, la distribución equitativa de los recursos y el respeto de la diversidad cultural. La dimensión económica se centra en la adecuada satisfacción de las necesidades materiales humanas, bajo distintas modalidades, adaptadas a las diversas culturas. La dimensión política, por su parte, está ligada a la necesidad de formas de gestión participativa, incluyendo la participación real de las comunidades en la diligencia de los recursos, como también en la toma de decisiones y formulación de políticas.

Planteado de una manera global, la sostenibilidad ecológica y la social son dos caras de una moneda, ya que, por una parte, la sostenibilidad social depende de la sostenibilidad ecológica: $\mathrm{Si}$ continuamos degradando la capacidad de la naturaleza de producir los servicios de los ecosistemas (filtración de las aguas, estabilización del clima, etc.) y de los recursos (alimentos, materiales), tanto los individuos como las naciones nos veremos afectados por crecientes presiones y aumento de conflictos, amenazas a la salud pública y a la seguridad personal. Por otra parte, la sostenibilidad ecológica depende de la social: con una población cada vez mayor que vive en un sistema social donde la satisfacción de las necesidades no se permite, es cada vez más difícil proteger el ambiente natural. Además, es necesario considerar que el comportamiento humano y la dinámica social que resulta es lo que subyace en el fondo de los problemas sociales y ecológicos (Onaindia-Olalde, 2007).

Una dimensión comúnmente olvidada en el marco del desarrollo sustentable es la dimensión psicológica: ésta se refiere a la satisfacción de necesidades que desembocan en el bienestar personal, las relaciones positivas, la felicidad, la restauración psicológica, la sensación de autoeficacia y la satisfacción personal, entre otras (Corral, 2012). Además, la dimensión psicológica de la sustentabilidad incluye los comportamientos por medio de los cuales las 
personas logran proteger su entorno social y físico. Lo anterior lleva implícito en el concepto de conducta sustentable, definida como el conjunto de acciones efectivas y deliberadas que tiene como finalidad el cuidado de los recursos naturales y socioculturales necesarios para garantizar el bienestar presente y futuro de la humanidad (Corral \& Pinheiro, 2004). Esta conducta, por supuesto, comprende acciones que llevan a la conservación del ambiente físico, pero también comportamientos encaminados a proteger a otros individuos y grupos sociales, especialmente los más vulnerables (Corral, 2012).

La conducta ambiental relevante ya no se considera sólo "pro-ambiental" o protectora del ambiente físico, sino "sustentable"; así lo indica una serie de dimensiones psicológicas que involucran la efectividad, la propensión al futuro, la deliberación, la austeridad y el altruismo, entre otras. Los determinantes de esta conducta abarcan variables (características individuales y situacionales, sistemas de intervención) que encaminan a las personas a actuar a favor del ambiente físico, de las sociedades presentes y futuras; y el efecto de esta conducta se da en las dimensiones económicas, sociales, ambientales y político/institucionales. La selección de los comportamientos relevantes (reutilización, reciclaje, ahorro de recursos, ayuda a otros, trato justo a las personas, etcétera) se da con base en el impacto de estos comportamientos en todas esas dimensiones. Lo anterior tiene implicaciones significativas en la manera de concebir, planear y diseñar la investigación social relacionada con la sostenibilidad. En esta nueva acepción no sólo se involucran nuevas variables en los instrumentos a aplicar, sino que, además, se emplean modelos ampliados y relaciones diferentes entre las variables (Corral, 2010). Esto obedece al hecho de que esas nuevas variables (económicas, sociales, políticas) operan en distintos niveles (exógenos intermedios) de la maraña de relaciones establecido entre los determinantes de la conducta sustentable y sus consecuencias (Corral \& Pinheiro, 2004).

La conducta sustentable para ser considerada como tal debe ser deliberada, es decir, realizada como un propósito y con la voluntad de llevarlo a cabo. Dicha conducta requiere de voluntad, conciencia y anticipación de los actos, de manera que estos procesos en conjunto permitan rectificar el curso de la acción comprendida y se adecuen a los cambios en las contingencias ambientales (Corral, 2010). Las conductas sustentables, de acuerdo con Corral (2010), se dividen en: comportamientos pro-ecológicos (cuidado de recursos naturales), frugales (disminución del consumo), altruistas (cuidado de otras personas), y equitativos (trato justo $\mathrm{y}$ sin sesgos hacia otras personas).

\section{En contacto con los principios ecológicos}

Otro posible camino para la sustentabilidad tiene relación con la ecología y sus principios, de manera que, al respetarlos, podamos satisfacer las necesidades actuales y futuras de la población mundial. Autores como Capra (1998) 
y Costa (2000) plantean que la supervivencia y la calidad de vida de la humanidad tienen estrecha conexión con nuestra "alfabetización ecológica", la cual se refiere a la comprensión de los principios ecológicos y su adaptación a las comunidades humanas teniendo en vista su sostenibilidad.

Dichos principios básicos de la ecología son: la interdependencia, el reciclaje, el flujo de la energía del sol, la asociación, la flexibilidad, la diversidad, la co-evolución, y como consecuencia de ellos, la sostenibilidad (Capra \& Pauli, 1995).

Posteriormente, Capra (1998) expuso una lista de principios de organización que identifica como principios básicos de la ecología, a fin de transportarlos a la sociedad y emplearlos como guía de construcción de comunidades humanas sustentables. El primer principio que menciona es la interdependencia, ésta se basa en la premisa de que todos los miembros de la comunidad ecológica están conectados en una amplia y compleja red de relaciones: la trama de la vida; comprender la interdependencia ecológica significa entender sus relaciones, toda vez que desde el punto de vista sistémico las interacciones entre las partes son tan o más importantes que las propias partes del todo. El segundo principio es la ciclicidad: al ser abiertos los sistemas en la naturaleza, los nutrientes son reciclados, por lo cual no hay producción de residuos; sin embargo, en las comunidades humanas el sistema productivo es lineal, con insumos que se extraen de esos sistemas, aunque sin reciclar los desechos. La asociación, es decir, la tendencia a establecer vínculos de cooperación, pero también de competencia, compone el tercero de los principios. El cuarto es la flexibilidad, o bien, la característica esencial de las comunidades sustentables que constituye un punto de equilibrio después de un período de cambios en las condiciones ambientales. Finalmente, tenemos el principio de la diversidad, que implica la presencia de diferencias (múltiples especies, diferencias genéticas e individuales) en la constitución de los ecosistemas, íntimamente ligado a la estructura en red del sistema. Un ecosistema diverso también será resiliente, pues posee muchas especies que superponen funciones ecológicas que pueden ser parcialmente sustituidas, en caso de que la red se deshaga.

Corral, Frías y García (2010), al postular la idea de la conducta sustentable como un constructo que involucra prácticas proecológicas y prosociales, afirman que el desarrollo que se mantiene a sí mismo busca la coherencia con los principios ecológicos, de modo que las reglas básicas de funcionamiento de los ecosistemas tienen que ser respetadas para promover el desarrollo humano y los comportamientos acordes con tal postulado. Estas reglas también incluyen los principios de interdependencia, diversidad, ciclicidad, flexibilidad, asociación y sustentabilidad, que son aplicables tanto a los ecosistemas naturales como a los humanos. 


\section{Los principios ecológicos, la alfabetización y la sintonía ecológica}

Para muchos autores la gran cantidad de problemas ambientales por la que atraviesa nuestro planeta puede tener su origen en la visión antropocéntrica que tiene el ser humano de su interacción con el medio ambiente. El término Antropocentrismo fue acuñado en 1860 en medio del debate sobre la teoría de la evolución darwinista, para indicar que los humanos son el centro del universo. "Antropocentrismo" significa "centrado en el hombre"; bajo esta óptica, el ser humano es un fin y la naturaleza con sus especies son un medio. El humano tiene deberes indirectos con la naturaleza en la medida que ésta le proporcione bienestar (Toca, 2011). El antropocentrismo considera a los seres humanos como la forma de vida más importante; otras formas lo serían sólo en la medida que impactaran o pudieran ser útiles a hombres y mujeres (Campbell, 1983; Kortenkamp \& Moore, 2001). Para Dunlap y Van Liere (1978), el antropocentrismo se caracteriza por realzar aquellos valores en donde el hombre está por encima de la naturaleza y puede servirse de ella sin ningún límite.

El antropocentrismo y la dominación de la naturaleza por el ser humano son las características más comunes de la civilización occidental. Estas actitudes, junto con los mecanismos económico-sociales de la sociedad occidental, han conducido a una profunda crisis ecológica. Los seres humanos se consideran superiores a la naturaleza. Según el sistema de valores de la civilización occidental, la naturaleza tiene que ser conquistada, dominada y manejada para satisfacer las necesidades de los seres humanos. Sin embargo, cuando el ser humano ignora los procesos naturales, y se enajena del mundo natural, empieza su propia destrucción (Jacorzynski, 2004).

Dunlap y Van Liere (1978) introdujeron una visión del mundo a la que llamaron Paradigma Social Dominante (PSD), el cual hace referencia a la visión antropocéntrica que guio al mundo occidental hasta los años sesenta del siglo XX. Este paradigma concibe a los humanos como una especie excepcional a lo cual no se aplican las reglas de interdependencia que rigen a los ecosistemas de la Tierra.

La ecología superficial es antropocéntrica, es decir, está centrada en el ser humano. Ve a éste por encima o aparte de la naturaleza, como fuente de todo valor, y le da a aquélla un valor únicamente instrumental (de uso). Por otro lado, opuesta a la ecología superficial surge la ecología profunda, la cual no separa a los humanos ni a ninguna otra especie o cosa del entorno natural. Concibe el mundo no como una colección de objetos aislados, sino como una red de fenómenos fundamentalmente interconectados e interdependientes. La ecología profunda reconoce el valor intrínseco de todos los seres vivos y concibe a los humanos como una mera hebra de la trama de la vida (Capra, 1998).

La ecología profunda nace en oposición a la ecología superficial, no es sino la lucha contra la contaminación y el agotamiento de los recursos, 
con el objetivo central de asegurar la salud y el enriquecimiento de las personas en los países desarrollados (Naess, 1973). Esta ecología considera a los enfoques ambientales centrados en los seres humanos como superficiales y arrogantes. La ecología superficial, entonces, entiende el ambiente como un instrumento para ser usado para beneficio de los humanos, mientras que la ecología profunda lo ve como fin y como medio de toda actividad humana. Esto significa que toda forma de vida tiene el mismo valor y no puede ser valorada de manera diferente, como "buena" o "mala" cuando se compara con otras formas de vida. Otro principio de dicha ecología es que no existen límites entre el ser humano y el ambiente, porque toda la vida se valora igual; cualquier división entre las formas de vida, incluyendo la humana, es ecología superficial (Rozzi, 2007).

En el marco de la ecología profunda, del reconocimiento de la interdependencia y del valor de todos los seres vivos y de la reconexión de los seres humanos con los procesos naturales surge la noción de "Alfabetización ecológica". Capra (1998) sostiene que podemos y debemos aprender de los ecosistemas la manera de vivir sustentablemente. A lo largo de más de tres mil millones de años de evolución, los ecosistemas del planeta se han organizado de formas sutiles y complejas para maximizar su sostenibilidad. Esta sabiduría natural es la esencia de la alfabetización ecológica. El autor argumenta que basándonos en la comprensión de los ecosistemas podemos formular una serie de principios de organización identificables como básicos para la ecología, que nos sirvan de líneas maestras sobre las cuales edificar comunidades humanas sustentables. Además de lo anterior, Corral (2010) sugiere que todos los principios ecológicos tienen su correspondiente dimensión psicológica, la cual puede (y debe) ser considerada cuando se buscan respuestas sustentables acerca de los dilemas ambientales.

Capra (1998) afirma que la supervivencia de la humanidad dependerá de la alfabetización ecológica, es decir, de la capacidad de comprender los principios de ecología y vivir en consecuencia. Para Pomier (2002), la esencia de la alfabetización ecológica se encuentra en el aprendizaje de los principios básicos de la ecología para que éstos operen como referencias morales del ser humano. Por su parte, Orr (1992) la define como la habilidad de entender el sistema natural que hace posible la vida en la tierra, para así comprender los principios detrás del funcionamiento de los ecosistemas y usar estos principios para crear futuros sustentables. Según Kramer (2003), la alfabetización ecológica significa conocer los principios básicos de la ecología y de las ciencias que la sostienen (química, física, biología, geología), así como desarrollar un espíritu que cuestione (en un mundo dominado por los mensajes mediáticos) las verdades aparentes, las sentencias inmutables y las opiniones establecidas.

A pesar de la significativa contribución que el concepto de Alfabetización Ecológica hace al de desarrollo sustentable, los autores del 
presente escrito detectamos la carencia, dentro del concepto, de un aspecto fundamental de la experiencia psicológica: el componente valorativo. La alfabetización ecológica enfatiza la parte cognitiva y racional (conocimiento, pensamiento crítico, moralidad) del contacto con los principios ecológicos; sin embargo, desatiende los motivos que llevan a las personas a actuar según las valoraciones desprendidas de sus emociones, afectos y afinidades con los objetos con los cuales interactúan (Vining \& Ebreo, 2002). Lo anterior significa que, para actuar en concordancia con los principios ecológicos, es necesario conocerlos, perotambién desplegar afinidad por ellos (es decir, valorarlos positivamente), para después aplicarlos (comportarse de manera sustentable). Nuestra idea de sintonía humana con los principios ecológicos (SHPE) implicaría, entonces, la existencia de una predisposición humana a conocer, valorar y aplicar esos principios. Para fundamentar esa idea, procederemos a revisar la literatura relevante, con el fin de encontrar indicios que apoyen la hipótesis de la existencia de la SHPE.

A continuación se describirán, con mayor detalle, cada uno de los principios ecológicos, y se explicará su relación con el desarrollo sustentable y la manera en que se podrían abordar estos principios desde la psicología. La pregunta que nos guía es si la mente humana se encuentra en sintonía con dichos principios. De ser así, se contaría con una plataforma de disposicionalidad psicológica (la SHPE) que serviría para encaminar a las personas hacia el desarrollo de estilos de vida más sustentables.

\section{Interdependencia}

El principio de interdependencia establece que todos los miembros de una comunidad ecológica se hallan interconectados en una vasta e intricada red de relaciones y considera que el éxito de toda la comunidad depende no sólo de la calidad de sus individuos, sino de la calidad de la comunidad como un todo; más aún: de la calidad de las relaciones que se establecen entre ellos, en particular de las conexiones de cooperación. En este sentido, una comunidad humana sustentable es consciente de las múltiples relaciones entre sus miembros (Capra, 1998). La interdependencia es la naturaleza de todas las relaciones ecológicas. El éxito de todo el sistema depende del éxito de sus miembros individuales, mientras que el éxito de cada miembro depende del éxito del sistema como un todo (Capra \& Pauli, 1995).

Sfeir-Younis (2009) asegura que el desarrollo sustentable es esencialmente una toma de conciencia de nuestras distintas formas de interdependencias, sean estas interespaciales o intertemporales. Pero la interdependencia (como tal, y dondequiera que ésta se manifiesta) es un estado del Ser. Es una experiencia externa e interna. Algunas personas son más sensibles a este estado o tienen un nivel de autorrealización más alto. Cuando la sensibilidad es muy baja, entonces el comportamiento humano es tal que se traduce fuertemente en destrucción 
ambiental. Este autor sostiene, además, que somos totalmente interdependientes y que debemos, por medio de la educación, introducir la idea de que vivimos en un mundo colectivo, interdependiente en su totalidad.

La interdependencia implica un intercambio que es relacional y dependiente en las relaciones de subordinación recíproca humana y comunitaria. Esto significa que existe adaptación y sostenibilidad como procesos continuos (Sisaye, 2012). Según Gómez del Campo (1999), el principio de interdependencia sostiene que la relación entre los elementos de un ecosistema (personas, roles, ambientes y reglas) es tal que, cuando ocurre un cambio en cualquiera de ellos, se alteran los otros. Lo anterior es apoyado por Nelson y Prilleltensky (2005), para quienes el principio de interdependencia significa que las diferentes partes de un ecosistema están interconectadas y que los cambios en cualquier parte del sistema tendrán un efecto dominó que impactará en otras partes del mismo.

Ahora bien, con respecto a la pregunta acerca de si los seres humanos entran en contacto (se sintonizan) con la cualidad de la interdependencia, ésta parece tener una respuesta positiva. Aparte de las reflexiones de Sfeir-Younis (2009), anteriormente comentadas, existe evidencia de investigación empírica que demuestran la existencia de un sistema de creencias contenida en una visión holística e interdependiente del mundo. Este sistema, llamado el Nuevo Paradigma de la Interdependencia Humana (NPIH, Corral,
Carrus, Bonnes, Moser, \& Sinha, 2008), plantea que el ecocentrismo y el antropocentrismo pueden combinarse para generar creencias más generales en las cuales se acepte y valore que la satisfacción de las necesidades humanas sea perfectamente compatible con el cuidado del entorno. Es decir, esta visión asume que las personas y su ambiente se necesitan entre sí (los primeros requieren de los recursos naturales para sobrevivir y el ambiente necesita de las personas para su conservación). El NPIH considera que las personas interdependen con la naturaleza y sus recursos y que los ecosistemas biológicos y culturales del presente interdependen con los del futuro (Corral, 2010). De acuerdo con los resultados de algunas investigaciones, el NPIH predice la ejecución de conductas protectoras del ambiente (Corral et al., 2008; Hernández, Suárez, Corral, \& Hess, 2012).

\section{Diversidad}

Otro de los principios ecológicos es la diversidad, entendida como el rango de variación o variedad que existe en un conjunto de atributos. La diversidad biológica se refiere, en consecuencia, la variabilidad que existe en el mundo vivo, es decir, en el seno de los escenarios en los cuales se desarrollan los individuos, y entre ellos mismos; este sentido amplio identifica a la biodiversidad con la vida en el mundo (Izca, 2004).

La integridad de los ecosistemas depende de la variedad de sus componentes, de tal manera que si uno falta puede ser compensado por los 
elementos restantes. Un ecosistema más diverso es más resiliente que un ecosistema menos diversificado (Pradhan, 2006).

La parte biótica del ecosistema la conforman los organismos, que constituyen la diversidad biológica o biodiversidad. La biodiversidad es la variedad de formas de vida sobre la Tierra, pero, además, desde el punto de vista de la ecología, el concepto comprende la heterogeneidad de interacciones entre las especies y su ambiente inmediato, para formar un ecosistema. Se distinguen habitualmente tres niveles en dicha variedad: genética o diversidad intraespecífica, consistente en la diversidad de genes; diversidad específica, entendida como diversidad de especies; y la diversidad ecosistémica, que se refiere a la diversidad de las comunidades biológicas cuya suma integrada constituye la biosfera (Onaindia-Olalde, 2007).

La diversidad ecológica es uno de los indicadores fundamentales de la integridad de un ecosistema. Un sistema o una región con muchas especies y diversidad genética $-\mathrm{y}$, por lo tanto, complejas redes de interacciones entre sus componentes- es mucho más fuerte y más capaz de adaptarse a los cambios o hacer frente a los factores de estrés. Un sistema con poca diversidad está menos preparado para adaptarse y por ende tiene una mayor probabilidad de no ser favorecido en la selección natural (Krebs, 2001).

Para Capra y Pauli (1995), la diversidad significa muchas relaciones diferentes, $\mathrm{y}$ múltiples enfoques para enfrentar el mismo problema. Una comunidad diversa es una comunidad resiliente, capaz de adaptarse con facilidad a las situaciones cambiantes. La pérdida de la biodiversidad, es decir, la pérdida diaria de las especies, es a la larga uno de nuestros más graves problemas ambientales globales. La diversidad natural proporciona una fuente de exploración y descubrimiento; el ingenio humano y la tecnología no pueden remplazar nunca el desafío de una vida en la naturaleza.

Kellert (1997) sostiene que la sociedad contemporánea con frecuencia falla al reconocer la importancia de mantener lazos saludables y ricos con la diversidad natural; además, argumenta que la calidad de vida de las personas depende de la riqueza de sus conexiones con la diversidad natural.

La perpetuación de la biodiversidad es una razón citada ampliamente para llevar a cabo la restauración ecológica. La preferencia por la conservación de la biodiversidad local es un valor apreciado, no sólo entre los biólogos y ecologistas, sino en gran parte del sector público en muchos países y culturas. Entre los ejemplos más conocidos de restauración dedicados al fomento de la biodiversidad se encuentran aquellos que pretenden beneficiar a las especies raras y en peligro de extinción (Clewell \& Aronson, 2006).

En los ecosistemas, el papel de la diversidad tiene íntima conexión con la estructura de red del sistema. Un ecosistema diverso también será elástico, porque contiene muchas especies con funciones ecológicas que se traslapan y pueden 
remplazarse parcialmente entre sí. Cuando una perturbación severa destruye una especie en particular, de modo que se rompe un eslabón de la red, una comunidad con diversidad podrá sobrevivir y reorganizarse porque otros eslabones en la red pueden cumplir por lo menos en parte la función de la especie destruida. En otras palabras, cuanto más complejo es el conjunto y su patrón de interconexiones, más elástico será (Sánchez, Guerrero, \& Castellanos, 2005).

Desde la perspectiva de la diversidad biocultural, un mundo sustentable es un mundo en el que no existe sólo diversidad biológica, sino además diversidad cultural y lingüística, como componentes críticos de la red de la vida y los factores que contribuyen a la vitalidad, la organización y la capacidad de resiliencia de los ecosistemas que sustentan la vida (Harmon, 2002).

Desde una perspectiva ecológica, la diversidad biológica y la cultural son una respuesta natural a las diferencias climáticas y geográficas a través de la superficie de la Tierra, ya que ambas han evolucionado juntas (Coleman, 1994).

Cuando hablamos de diversidad social nos referimos a la variedad de identidades, experiencias y oportunidades que se manifiestan en los diferentes grupos y personas. Las dimensiones de la diversidad incluyen (pero no se limitan): la edad, el origen étnico, el género, las capacidades o cualidades físicas, raza, orientación sexual, el nivel educativo, la situación geográfica, el ingreso, estado civil, estado de paternidad, tipo de religión, y experiencia laboral (Bound, 2006).

Gadgil (1987) observó que la diversidad cultural humana y la biológica van de la mano en tanto prerrequisitos para la sostenibilidad a largo plazo. La diversidad social es un elemento típicamente relacionado con la sostenibilidad, en principio porque reduce las consecuencias problemáticas derivadas de la segregación y concentración de grupos marginales, pero también porque mejora el número y variedad de oportunidades que la comunidad ofrece, además de facilitar la continuidad en la provisión de servicios (Montero, Bosque \& Romero, 2008).

Hablando de los factores psicológicos de sintonía con los principios ecológicos, la afinidad por la diversidad $(A D)$ es la correspondiente dimensión de sintonía humana de la diversidad como principio ecológico. De acuerdo con la investigación emprendida hasta ahora, esta afinidad reflejaría un gusto por la variedad biológica (plantas y animales), física (climas, geografías), y social (culturas, religiones, sexos y orientaciones sexuales, edades, inclinaciones políticas) con las que un individuo entra en contacto (Corral, Tapia, Fraijo, Mireles, \& Márquez, 2008). Además, la afinidad por la diversidad es, conceptualmente hablando, parte de la idea de sostenibilidad (Kellert, 1997). Corral, Bonnes, Tapia, Fraijo, Frías y Carrus (2009) encontraron que mientras mayor era la $\mathrm{AD}$ de las personas, más notorio era también su esfuerzo por cuidar el ambiente social y natural. 


\section{Asociación}

Uno de los principios ecológicos básicos que hemos mencionado es la asociación: la cooperación y la competencia son sus características definitorias. La asociación es fundamental en las comunidades sustentables y se define como una tendencia a la formación de lazos y uniones para lograr un fin compartido, teniendo en cuenta que la cooperación es un requisito para la calidad de vida. Conciliar esta asociación con el proceso de transformación y desarrollo llevará a la evolución, debido a que los individuos evolucionan a través de una asociación confiable (Costa, 2000). Además, la asociación es un criterio esencial para la operatividad de la sostenibilidad. Los intercambios de energía y recursos están sustentados por la cooperación. Establecer asociaciones garantiza la calidad de vida y de co-evolución (Dimauro \& De Manuel, 2010). Al respecto, Pol (2002) encontró que la gente orientada a la sostenibilidad es también cooperativa y ayuda a otros que se encuentran en necesidad.

Dicho principio es una necesidad biológica y de subsistencia, no solo a nivel ecológico o individual, sino a nivel colectivo. Los estados deben cooperar entre sí para erradicar la pobreza, como requisito indispensable del desarrollo sustentable; para proteger la integridad del ecosistema de la Tierra; para reforzar la creación de capacidades endógenas; para lograr el desarrollo sustentable y abordar los problemas de degradación ambiental. La defensa del ecosistema, en efecto, obliga a la cooperación global, pues, de lo contrario, cualquier esfuerzo en tal sentido sería en vano (Cafferatta, 2004). Al interior de las comunidades, es notorio que el esfuerzo de cooperación para la conservación ambiental entre los ciudadanos es superior al empeño individual (Corral, 2010).

Mientras la economía enfatiza la competencia, la expansión y la dominación, la ecología pone acento en la cooperación, la conservación y la asociación, es decir, privilegia un patrón de pensamiento y valores integrativos (Capra, 1998).

Sin embargo, debe reconocerse que, a la par de las relaciones de asociación, existen las de competencia, como propiedad necesaria. La competencia se define como la relación que entablan dos organismos cuyo requerimiento de recurso es el mismo y tratan cada cual de conseguirlo. Los organismos que compiten pueden ser de la misma o de diferente especie. Ocasionalmente, esta interacción lleva a que los organismos competidores resulten perjudicados (Valverde, Meave, Carabias, \& Cano, 2005). La competencia es uno de los fenómenos más generalizados, observándose en todos los niveles de la vida en la lucha por la supervivencia. Dentro de un hábitat determinado, algunas especias logran imponerse a otras debido a su mayor vigor y resistencia (López et al., 2006). Según Peñalver, Pargas y Aguilera (2000), la competencia es una especie de mecanismo de carácter espontáneo que regula la relación entre el número de habitantes y los recursos del hábitat. A través de la competencia, las 
diferentes especies encuentran un lugar en el medio ambiente, ya que la competencia genera una división del trabajo basada en una economía natural. Este equilibrio no es permanente, puede verse obstruido por el aumento del número de habitantes, o por la invasión del hábitat por parte de una especie extranjera; situación que podría superarse mediante una emigración de la población sobrante, o mediante un nuevo reparto de los recursos del habitar a través de la competencia.

En una sociedad humana, la competencia encaminada a la sostenibilidad puede implicar el desarrollo de esfuerzos, ya sea individuales o grupales, para lograr antes que otros y de manera más efectiva, un objetivo, por ejemplo, procesos de ahorro energético, sistemas de protección a ecosistemas, mecanismos de protección de recursos naturales, etcétera. La competencia tendría, en estos casos, resultados positivos en la innovación para la resolución de problemas ambientales.

\section{Flexibilidad}

La flexibilidad se manifiesta en el hecho de que la estructura de la red de un ecosistema no es rígida, por el contrario, está en constante fluctuación. Cuando cambian las condiciones ambientales, por ejemplo, un verano inusualmente cálido, se perturba un eslabón del ciclo ecológico; entonces, el ciclo entero actúa como auto-regulador y pronto la situación regresa al equilibrio. Dado que estas perturbaciones ambientales ocurren todo el tiempo, las variables de un ciclo ecológico (suministro de nutrientes, la densidad de población, etcétera) se someten a continuas fluctuaciones interdependientes. Estas fluctuaciones representan la flexibilidad de ecosistema. Mientras más variables se mantengan fluctuando, el sistema es más dinámico, mayor es su flexibilidad y mayor es su capacidad para adaptarse a las condiciones ambientales cambiantes (Capra \& Pauli, 1995).

La flexibilidad es el criterio que determina cuándo un ecosistema se encuentre preparado para adaptarse a las condiciones ambientales. La multifuncionalidad ofrece más oportunidades para la práctica de la sostenibilidad que la rigidez y la superespecialización a la hora de abordar la complejidad. Cuando un desafío presentado por el medio persiste, los sistemas abiertos se desmontan y se adaptan, reorganizándose en mejores y más complejas formas, evolucionando (Dimauro \& De Manuel, 2010). La tensión temporal es un aspecto esencial en la vida, sin embargo, la tensión prolongada es dañina y destructiva para los sistemas (Capra, 1998).

A nivel psicológico, la flexibilidad se define como la capacidad de ajustar los pensamientos, sentimientos y conducta a situaciones $y$ condiciones cambiantes, no predecibles y/o no familiares. Las personas flexibles reaccionan al cambio sin rigidez, con agilidad y energía; suelen estar abiertas y son tolerantes frente a posturas, ideas y creencias diferentes de las propias y están dispuestas a cambiar si están equivocadas (Corral, 2012). La versatilidad, uno de los componentes esenciales de la flexibilidad 
psicológica, resalta en la idea de la competencia pro-ambiental (Corral, 2010). Fraijo, Corral, Tapia y González (2010) demuestran que los individuos más versátiles (flexibles) en la búsqueda de soluciones a problemas ambientales, son más competentes, y esto los lleva a actuar de manera sustentable, con más facilidad.

\section{Ciclicidad}

Según Capra y Pauli (1995), la interdependencia entre los miembros de un ecosistema involucra el intercambio de energía y recursos en ciclos continuos. Las comunidades de organismos han evolucionado durante miles de millones de años, de forma constante usando y reciclando las mismas moléculas de los minerales, el agua y el aire.

El conflicto actual entre la empresa y la naturaleza o entre la economía y la ecología, se debe principalmente al hecho de que la naturaleza es cíclica, mientras que la mayoría de los sistemas industriales son lineales, tomando la energía y los recursos de la tierra, transformándolos en desperdicio, desechando los residuos y por último, tirando los productos también, después de que han sido usados (Capra \& Pauli, 1995).

Un ecosistema está en equilibrio cuando sus ciclos se cumplen con normalidad, restaurando los recursos utilizados; si el fenómeno no se produce en estas condiciones el equilibrio se altera, rompiéndose el orden del proceso e iniciándose su deterioro. El equilibrio ecológico se produce entre las especies vivas y el ambiente total en que ellas habitan y del cual viven (San Martín, 1983).

En la naturaleza nada se pierde y todo lo que es desechado por una especie es aprovechado por otra. La conservación de los recursos energéticos y materiales destinados al abastecimiento de las ciudades debe ser realizada a través de procesos más eficientes y respetuosos con la naturaleza, cerrando los ciclos de materia y energía y considerando los flujos de inicio (fuentes) hasta el final (residuos) (Dimauro \& De Manuel, 2010). Los patrones sustentables de producción y consumo deben ser cíclicos, imitando los procesos de los ecosistemas. Para lograr estos patrones cíclicos, tenemos que rediseñar fundamentalmente nuestra economía (Capra \& Pauli, 1995).

Aunque no se detectan investigaciones que muestren, de manera explícita, la sintonía psicológica con el principio de la ciclicidad, la existencia de sectores cada vez mayores de población que aceptan y se involucran en la práctica de acciones como el reciclaje, el reuso de productos, el compostaje, y la puesta en marcha de procesos de reutilización de productos en sistemas industriales (Corral, 2010), sugiere la existencia de un cierto nivel de sintonía humana con este principio. 


\section{Discusión}

Como lo sugiere la revisión antes emprendida, los principios ecológicos propios de los ecosistemas y deseables en las comunidades sustentables se pudieran extender al nivel personal. Las evidencias parecen mostrar que todos los principios ecológicos tienen su correspondiente dimensión psicológica, que debe ser considerada cuando se buscan respuestas sustentables para los dilemas ambientales (Corral, Frías, \& García, 2010).

Como se observó en apartados anteriores, existen diversas teorías que afirman la presencia de similitudes entre las comunidades humanas y los ecosistemas, y que de estos últimos podemos aprender cómo vivir sustentablemente. No obstante, a nivel conductual esta posible relación entre la práctica de conductas sustentables y el vivir de acuerdo con los principios ecológicos ha sido muy poco estudiada, por tanto, las indagaciones acerca de tal relación podrían ser importantes para promover las conductas pro ambientales y pro sociales. La sintonía con los principios ecológicos la podemos observar en el grado de alfabetización y afinidad ecológica que presentan las personas, es decir, la capacidad para comprender y valorar los principios de la ecología (flexibilidad, diversidad, interdependencia, ciclicidad y asociación) y actuar de acuerdo con ellos. Así, a mayor sintonía con los principios ecológico mayor será la práctica de conductas sustentables.
Empero, a pesar de los indicios plausibles de esa descripción, los estudios empíricos empleados para demostrar dicha relación son aislados. Se requiere investigación adicional que demuestre la existencia de un factor de sintonía humana con los principios ecológicos. Dicho factor tendría como indicadores el acuerdo con y la evaluación positiva de los principios ecológicos. Además, la sintonía con esos principios se manifestaría en relaciones significativas entre ese factor y las conductas sustentables (altruismo, austeridad, conductas proecológicas y equitativas) mencionados previamente. Lo anterior significaría que una persona sintonizada con los principios ecológicos no solamente los conocería, aceptaría y valoraría de forma positiva, sino que, además, los practicaría.

De probarse esta relación, pudieran instrumentarse programas en los que se utilizaría el análisis de los principios que rigen a los ecosistemas. En el campo de la educación ambiental, sería posible orientar los contenidos hacia la práctica de acciones sustentables con base en las guías de la alfabetización y la sintonía con los principios ecológicos. De acuerdo con Montoya y Russo (2007), existen muchas posibilidades de revertir el impacto de la degradación de los recursos naturales si se aplican los principios básicos de alfabetización ecológica como una herramienta para la educación ambiental. Nuestra idea es que la alfabetización ecológica, entendida como la 
adquisición y uso de conocimientos referidos a la ecología, es necesaria pero no suficiente, ya que es necesario agregar el componente afectivovalorativo que lleva a las personas a actuar: la afinidad, el gusto y la motivación derivados del conocimiento y uso de los principios ecológicos. La SHPE incorpora, como lo planteamos arriba, las tres dimensiones esenciales: cognitivas (conocimiento), valorativas (afectividad) y conductuales (práctica), por lo cual, consideramos, se constituiría en un factor determinante de la actuación sustentable.

Por último, en caso de confirmarse nuestras hipótesis, este potencial hallazgo pudiera ayudar a orientar la actual vida de consumo de las personas a patrones de vida y consumo más sustentables, basados en los principios ecológicos. También ayudaría a los seres humanos en su búsqueda de bienestar personal y de calidad del entorno socio-físico en el que se desenvuelven, al aproximarlos con la naturaleza, la comunidad, y armonizando sus necesidades con las de la biosfera planetaria.

\section{Referencias}

Bound, K. (2006). Social diversity and cities. Recuperado de http://www.britishcouncil. org/networkeffect-amst.pdf.

Brundtland, G. H. (1987). Our common future. Oxford, UK: Oxford University Press.

Cafferatta, N. A. (2004). Introducción al derecho ambiental. México: Secretaría del Medio Ambiente y Recursos Naturales.
Campbell, E. K. (1983). Beyond anthropocentrism. Journal of the History of the Behavioral Sciences, 19(1), 54-67.

Capra, F. (1998). La trama de la vida, Barcelona: Anagrama.

Capra, F., \& Pauli, G. A. (1995). Steering business toward sustainability. Tokyo: United Nations University Press.

Clewell, A. F., \& Aronson, J. (2006). Motivations for the Restoration of Ecosystems. Conservation Biology, 20(2), 420-428.

Coleman, D. A. (1994). Ecopolitics: Building a green society. New Brunswick, NJ: Rutgers University Press.

Corral, V. (2010). Psicología de la sustentabilidad: Un análisis de lo que nos hace pro ecológicos y pro sociales. México, DF: Trillas.

Corral, V. (2012). Sustentabilidad y psicologia postiva: Una visión optimista de las conductas proambientales y prosociales. México: Manual Moderno.

Corral, V., Bonnes, M., Tapia, C., Fraijo, B., Frías, M., \& Carrus, G. (2009). Correlates of pro-sustainability orientation: The affinity towards diversity. Journal of Environmental Psychology, 29, 34-43.

Corral, V., Carrus, G., Bonnes, M., Moser, G., \& Sinha, J. (2008). Environmental beliefs and endorsement of Sustainable Development principles in water conservation: Towards a New Human Interdependence Paradigm scale. Environment \& Behavior, 40, 703-725.

Corral, V., Frías, M., \& García, C. (2010). Introduction to the psychological dimensions 
of sustainability. En V. Corral, C. García \& M. Frías (Eds.), Psychological Approaches to Sustainability. New York: Nova Science Publishers.

Corral,V.,\&Pinheiro, V.(2004). Aproximaciones al estudio de la conducta sustentable. Medio Ambiente y Comportamiento Humano, 5, $1-26$.

Corral, V., Tapia, C., Fraijo, B., Mireles, J., \& Márquez, P. (2008). Orientación a la Sustentabilidad como Determinante de los estilos de vida sustentables: Un estudio con una muestra mexicana. Revista Mexicana de Psicología, 25, 313-327.

Costa, I. (2000). Principios ecológicos y calidad de vida. Revista Latino-Americana de Enfermagem, 8(4), 1-5.

Díaz, C., \& Spiaggi, E. (2007). Ruralidad y Desarrollo en le Región Pampeana: Estrategias para la sustentabilidad. Argentina, Rosario: UNR Editora.

Dimauro, G., \& De Manuel, E. (2010). Comunidades en transición: Hacia otras prácticas sostenibles en los ecosistemas urbanos. Revista Cidades, Comunidades e Territórios, 20, 1-21.

Dunlap, R. E., \& Van Liere, K. D. (1978). The New Environmental Paradigm. Journal of Environmental Education, 9, 10-19.

Fraijo, B., Corral, V., Tapia, C., \& González, D. (2010). Promoting pro-environmental competency. En V. Corral, C. García \& M. Frías (Eds.), Psychological Approaches to
Sustainability (pp. 225-246). New York: Nova Science Publishers.

Gadgil, M. (1987). Diversity: Cultural and ecological. Trends in Ecology, 2, 369-373.

Gómez, del Campo, J. (1999). Psicología de la comunidad: Perspectivas teóricas, modelos y aplicaciones, un punto de vista humanista. México, DF: Plaza y Valdes.

Gowdy, J. M., \& McDaniel, C. N. (1995). One world, one experiment: Addressing the biodiversity-economics conflict. Ecological Economics, 15(3), 181-192.

Harmon, D. (2002). In Light of Our Differences: How Diversity in Nature and Culture Makes Us Human. Washington, DC: Smithsonian Institution Press.

Hernández, B., Suárez, E., Corral, V., \& Hess, S. (2012). The Relationship between Social and Environmental Interdependence as an Explanation of Proenvironmental Behavior. Human Ecology Review, 19, 1-9.

Izca, J. (2004). Botánica. España, Madrid: McGraw-Hill.

Jacorzynski, W. (2004). Entre los sueños de la razón: Filosofía y antropología de las relaciones entre hombre y ambiente. México, DF: Centro de Investigaciones y Estudios Superiores en Antropología Social.

Kammerbauer, J. (2001). Las dimensiones de la sostenibilidad: Fundamentos ecológicos, modelos paradigmáticos y senderos. Interciencia, 26(8), 353-359. 
Kellert, S. R. (1997). Kinship to mastery: Biophilia in human evolution and development. Washington, DC: Island Press.

Kortenkamp, K., \& Moore, C. (2001). Ecocentrism and Anthropocentrism: Moral reasoning about ecological commons dilemmas. Journal of Environmental Psychology, 21(3), 261-272.

Kramer, F. (2003). Educación ambiental para el desarrollo sostenible. Madrid: Los Libros de la Catarata.

Krebs, C. J. (2001). Ecology: The experimental analysis of distribution and abundance. San Francisco: Benjamin Cummings.

López, A., Chagollan, F., Del Campo, M., García, R., Contreras, I., \& García, R. (2006). Ecología. México: Umbral.

Montero, J. Bosque, J., \& Romero, R. (2008). Cuantificación y cartografía de la sostenibilidad social a partir de tipologías urbanísticas. En L. Hernández \& J. Parreño (Eds.), Tecnologías de la Información Geográfica para el Desarrollo Territorial (pp. 76-91). España: Servicio de Publicaciones y Difusión Científica de la ULPGC.

Montoya, C., \& Russo, R. (2007). Ecoalfabetización: Una herramienta de educación ambiental. Revista Comunicación, agostodiciembre, 83-85.

Naess, A. (1973). The shallow and the deep, long-range ecology movement. Inquiry, 16, 95-100.

Nelson, G. B., \& Prilleltensky, I. (2005). Community psychology: In pursuit of liberation and well-being. Houndmills, Basingstoke, Hampshire: Palgrave Macmillan.

Onaindia-Olalde, M. (2007). Sostenibilidad ecológica. Forum de Sostenibilidad, 1, 3949.

Orr, D. W. (1992). Ecological Literacy: Education and the transition to a postmodern world. Albany: State University of New York Press.

Pradhan, S. K. (2006). Building resilience in local institution for natural resource management. Anand, India: Foundation for Ecological Security.

Pol, E. (2002). The theoretical background of the City-Identity-Sustainability Network. Environment \& Behavior, 34, 8-25.

Peñalver, L., Pargas, L., \& Aguilera, O. (2000). Pensar lo urbano: Teorías, mitos y movimientos. Venezuela: Universidad de los Andes.

Pomier, P. (2002). Determinismo biológico: El desafío de la alfabetización ecológica en la concepción de Fritjof Capra. Tópicos en Educación Ambiental, 4(11), 7-18.

Rozzi, R. (2007). Ecología superficial y profunda: Filosofía ecológica. Revista Ambiente y Desarrollo, 23, 102-105.

San Martín, H. (1983). Ecología humana y salud: El hombre y su ambiente. México, DF: Ediciones Científicas, La Prensa Médica Mexicana.

Sánchez, H., Guerrero, F., \& Castellanos, M. (2005). Ecología. México, Zapopan: Umbral Editorial. 
Sfeir-Younis, A. (2009). Educación, economía ambiental y espiritualidad. Revista de Estudios Sociales, 32, 241-254.

Sisaye, S. (2012). An ecological analysis of four competing approaches to sustainability development:Integration of industrial ecology and ecological anthropology literature. World Journal of Entrepreneurship, Management and Sustainable Development, 8(1), 18-35.

Toca, C. (2011). Las versiones del desarrollo sostenible. Sociedade e Cultura, 14, 195-204. Valverde, V. T., Meave, C. J. A., Carabias, L., \& Cano, S. Z. (2005). Ecología y medio ambiente. México: Pearson Educación.

Vining, J., \& Ebreo, A. (2002). Emerging theoretical and methodological perspectives on conservation behavior. En R.B. Bechtel \& A. Churchman (Eds.), Handbook of Environmental Psychology (pp. 541-558). New York: Wiley. 